\title{
An Order Reduction Method of System Dynamic Equations Based on Vector Bond Graph
}

\author{
Zhongshuang Wang ${ }^{1,}$, Meng Ren ${ }^{2, b}$ \\ ${ }^{1,2}$ School of Mechatronic Engineering, Qiqihar University ,Qiqihar, Heilongjiang, 161006, P.R. \\ China \\ a'Whsh1962@163.com
}

\begin{abstract}
Keywords: Vector bond graph, Dynamic modeling , Order reduction method, Causality, Mass spring system.

Abstract. In order to increase the efficiency of system dynamic modeling and simulation, the order reduction method of system dynamic equations based on vector bond graph is proposed. Based on system independent energy storage field, resistive field, source field and junction structure, the different unified formula for order reduction of system dynamic equation is derived. Thus, the different order reduction method can be selected by the need of practical problem. The proposed method is organized and very suitable for computer aide dynamic analysis. Based on this, the computer aided order reduction of system dynamic equations for a mass spring system is realized, and the validity of this procedure is illustrated.
\end{abstract}

\section{Introduction}

For modern mechanical system with high speed and accuracy, the dynamic analysis is very important to its optimum design and improvement of dynamic characteristics. Although different computer aided dynamic modeling and simulation procedures have been proposed[1,2], these procedures are only suitable for a single energy domain, such as strict mechanical one, and can not be used to deal with the problems of computer aided dynamic analysis of mechanical systems containing the coupling of multi-energy domains. Bond graphs[3] have potential applications in analyzing such complex systems because of their ability to describe the dynamics of interacting systems over a multi-energy domain in a unified manner. Besides this, they also have the following characteristics, such as the unification of graph and dynamics descriptions and organized manner of dynamic modeling. For some complex engineering systems, larger numbers of state variables result in larger numbers of system state space equations. Thus, the efficiency of system modeling and simulation is decreased. To solve above problem, the order reduction method of system dynamic equations should be studied further.

Based on scalar bond graphs, an order reduction procedure was proposed [4], and its validity is illustrated by the modeling and simulation of dynamics for a vehicle suspension system. Compared with scalar bond graph, vector bond graph contains more system information and is more suitable for modelling and simulation of complex systems because of its more concise representation manner $[5,6,7]$. However, how to realize the computer aided order reduction of system dynamic equations based on vector bond graph should be studied further. In what follows, the order reduction method of system dynamic equations based vector bond graph is described. By this procedure, the different formula of order reduction can be selected by the need of practical problems, and the computer aided order reduction of a mass spring system is realized.

\section{The unified formula of order reduction for system dynamic equations}

In Fig.1, a system vector bond graph can be divided into basic fields and junction structure[3]. Independent energy storage field consists of inertia element I and capacitance element $\mathrm{C}$ with integral causality, resistive field consists of resistance element $\mathrm{R}$, and source field contains effort source element Se and flow source element Sf. The input and output vectors are shown in Fig.1, where $X$ represents energy variable vector of independent storage energy field, $Z$ is the corresponding 
coenergy variable vector. $X=\left[\begin{array}{ll}p & q\end{array}\right]^{T}$, vector $p$ is system generalized momentum, $\mathrm{p}=\left[\begin{array}{lll}p_{1} & p_{2} \mathrm{~L} & p_{m}\end{array}\right]^{T}$, vector $\mathrm{q}$ is system generalized displacement vector, $\mathrm{q}=\left[\begin{array}{llll}q_{1} & q_{2} & \mathrm{~L} & q_{n}\end{array}\right]^{T}$. $\mathrm{D}_{\text {in }}$ and $\mathrm{D}_{\text {out }}$ represent input and output variable vectors in resistive field. $\mathrm{V}$ and $\mathrm{U}$ represent input and output variable vectors of source field respectively, where source output effort vector $\mathrm{U}_{e}=\left[\begin{array}{llll}S e_{1} & S e_{2} & \mathrm{~L} & S e_{s}\end{array}\right]^{T}$, and source output flow vector $\mathrm{U}_{f}=\left[\begin{array}{llll}S f_{1} & S f_{2} & \mathrm{~L} & S f_{r}\end{array}\right]^{T}$.

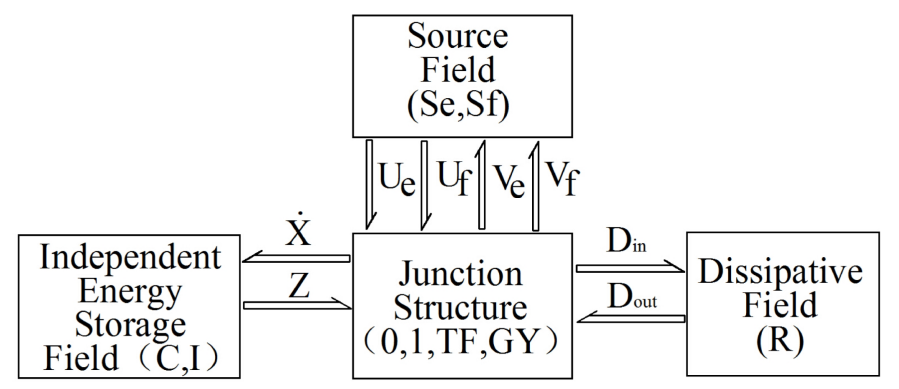

Fig. 1 The basic fields and junction structure of system

From the relations of input and output vectors of system basic fields and junction structure[3], the unified formula of system state space equations can be written as $X \mathcal{E}=A X+B U$, and can be further expressed as

$$
\left[\begin{array}{l}
\boldsymbol{\alpha} \\
q \boldsymbol{\phi}
\end{array}\right]=\left[\begin{array}{ll}
\mathrm{A}_{p p} & \mathrm{~A}_{p q} \\
\mathrm{~A}_{q p} & \mathrm{~A}_{q q}
\end{array}\right]\left[\begin{array}{l}
\mathrm{p} \\
\mathrm{q}
\end{array}\right]+\left[\begin{array}{ll}
\mathrm{B}_{p e} & \mathrm{~B}_{p f} \\
\mathrm{~B}_{q e} & \mathrm{~B}_{q f}
\end{array}\right]\left[\begin{array}{l}
\mathrm{U}_{e} \\
\mathrm{U}_{f}
\end{array}\right]
$$

where, $\mathrm{A}_{p p}, \mathrm{~A}_{p q}, \mathrm{~A}_{q p}$ and $\mathrm{A}_{q q}$ are $m \times m, m \times n, n \times m$, and $n \times n$ matrices, $\mathrm{B}_{p e}, \mathrm{~B}_{p f}, \mathrm{~B}_{q e}$ and $\mathrm{B}_{q f}$ are $m \times s, m \times r, n \times s$ and $n \times r$ matrices respectively

Eq. 1 can be further written as

$$
\begin{aligned}
& \alpha=\mathrm{A}_{p p} \mathrm{p}+\mathrm{A}_{p q} \mathrm{q}+\mathrm{B}_{p e} \mathrm{U}_{e}+\mathrm{B}_{p f} \mathrm{U}_{f} \\
& \phi \alpha=\mathrm{A}_{q p} \mathrm{p}+\mathrm{A}_{q q} \mathrm{q}+\mathrm{B}_{q e} \mathrm{U}_{e}+\mathrm{B}_{q f} \mathrm{U}_{f}
\end{aligned}
$$

By using differential operator sign $\mathrm{d}$, we can get $d \mathrm{q}=\phi \phi, d^{-1} \mathrm{q}=\int \mathrm{q} d t$. From Eq.3, we have

$$
\left[d-\mathrm{A}_{q q}\right] \mathrm{q}=\mathrm{A}_{q p} \mathrm{p}+\mathrm{B}_{q e} \mathrm{U}_{e}+\mathrm{B}_{q f} \mathrm{U}_{f}
$$

Let the dimension of vector $p$ and $q$ be equal (this limit can be eliminated at last), by the manipulation of Eq.2 and Eq.4, we have

$$
\left[d-\mathrm{A}_{q q}\right] \mathrm{A}_{p q}^{-1} \boldsymbol{\alpha}=\left[d-\mathrm{A}_{q q}\right] \mathrm{A}_{p q}^{-1}\left[\mathrm{~A}_{p p} \mathrm{p}+\mathrm{B}_{p e} \mathrm{U}_{e}+\mathrm{B}_{p f} \mathrm{U}_{f}\right]+\mathrm{A}_{q p} \mathrm{p}+\mathrm{B}_{q e} \mathrm{U}_{e}+\mathrm{B}_{q f} \mathrm{U}_{f}
$$

From Eq.5, we can get

$$
\mathrm{MX}_{I}^{\mathcal{\alpha}}+\mathrm{BX}_{I}^{\mathcal{\alpha}_{I}}+\mathrm{KX}_{I}=\mathrm{B}_{p e} \mathrm{U}_{e}+\mathrm{B}_{p f} \mathrm{U}_{f}+\mathrm{T}_{1} \int_{0}^{t} \mathrm{U}_{e} d t+\mathrm{T}_{2} \int_{0}^{t} \mathrm{U}_{f} d t
$$

where, $X_{I}$ represents displacement vector of inertia elements.

$$
\begin{aligned}
& \mathrm{M}=\operatorname{diag}\left(I_{1} \quad I_{2} \quad \mathrm{~L} \quad I_{n}\right), \mathrm{B}=\left(-\mathrm{A}_{p q} \mathrm{~A}_{q q} \mathrm{~A}_{p q}^{-1}-\mathrm{A}_{p p}\right) \mathrm{M}, \mathrm{K}=\left(\mathrm{A}_{p q} \mathrm{~A}_{q q} \mathrm{~A}_{p q}^{-1} \mathrm{~A}_{p p}-\mathrm{A}_{p q} \mathrm{~A}_{q p}\right) \mathrm{M} \\
& \mathrm{T}_{1}=\mathrm{A}_{p q} \mathrm{~B}_{q e}-\mathrm{A}_{p q} \mathrm{~A}_{q q} \mathrm{~A}_{p q}^{-1} \mathrm{~B}_{p e}, \mathrm{~T}_{2}=\mathrm{A}_{p q} \mathrm{~B}_{q f}-\mathrm{A}_{p q} \mathrm{~A}_{q q} \mathrm{~A}_{p q}^{-1} \mathrm{~B}_{p f}
\end{aligned}
$$

In the process of system modeling, describing system damping exactly is usually difficult. So the damping effect of resistive element in a system can be neglected temporarily, it can be added after finishing the whole process of system modeling. If the effect of resistive element in a system is 
neglected, the junction structure of system bond graph does not contain GY element, so that $A_{p p}=0$, $\mathrm{A}_{q q}=0$, Eq. 6 can be simplified as

$$
\mathrm{M} \frac{\mathrm{\alpha}}{I}+\mathrm{KX} \mathrm{B}_{I}=\mathrm{B}_{p e} \mathrm{U}_{e}+\mathrm{B}_{p f} \mathrm{U}_{f}+\mathrm{T}_{1} \int_{0}^{t} \mathrm{U}_{e} d t+\mathrm{T}_{2} \int_{0}^{t} \mathrm{U}_{f} d t
$$

where, $\mathrm{K}=-\mathrm{A}_{p q} \mathrm{~A}_{q p} \mathrm{M}, \mathrm{T}_{1}=\mathrm{A}_{p q} \mathrm{~B}_{q e}, \mathrm{~T}_{2}=\mathrm{A}_{p q} \mathrm{~B}_{q f}$. In this case, the limit that the dimension of vector $p$ and vector $q$ is equal can be eliminated.

When the dimension of vector $p$ is less than that of vector $q$, the equation number of Eq.7 is less than that of Eq.1. As a result, the displacement vector $X_{I}$ and flow vector $X_{I}^{\mathcal{E}}$ of inertia elements can be determined by Eq.7 directly. Thus, the efficiency of system modeling can be increased.

When the dimension of vector $p$ is more than that of vector $q$, another form of system dynamic equations can be obtained in like manner, and shown as Eq.8.

$$
\mathrm{A}_{1} \boldsymbol{q}+\mathrm{A}_{2} \mathrm{q}=\mathrm{WU}_{e}+\mathrm{W}_{2} \mathrm{U}_{f}+\mathrm{B}_{q e} \boldsymbol{\psi}_{e}+\mathrm{B}_{q f} \boldsymbol{\psi}_{f}^{\alpha}
$$

wh h r e, $\quad \mathrm{A}_{1}=-\mathrm{A}_{q p} \mathrm{~A}_{p p} \mathrm{~A}_{q p}^{-1}-\mathrm{A}_{q q}, \mathrm{~A}_{2}=\mathrm{A}_{q p} \mathrm{~A}_{p p} \mathrm{~A}_{q p}^{-1} \mathrm{~A}_{q q}-\mathrm{A}_{q p} \mathrm{~A}_{p q}, \mathrm{~W}=-\mathrm{A}_{q p} \mathrm{~A}_{p p} \mathrm{~A}_{q p}^{-1} \mathrm{~B}_{p e}+\mathrm{A}_{q p} \mathrm{~B}_{p e}, \mathrm{~W}_{2}=\mathrm{A}_{q p} \mathrm{~B}_{p f}$ $-\mathrm{A}_{q p} \mathrm{~A}_{p p} \mathrm{~A}_{q p}^{-1} \mathrm{~B}_{q f}$.

In like manner, the resistive element can be neglected temporarily. Thus, $A_{p p}=0, A_{q q}=0$. From Eq.8, we have

$$
\mathrm{A}_{2} \mathrm{q}=\mathrm{WU}_{e}+\mathrm{WU}_{2}+\mathrm{B}_{q e} \boldsymbol{U}_{e}+\mathrm{B}_{q f} \boldsymbol{U}_{f}^{\alpha}
$$

where, $\mathrm{A}_{2}=-\mathrm{A}_{q p} \mathrm{~A}_{p q}, \mathrm{~W}=\mathrm{A}_{q p} \mathrm{~B}_{p e}, \mathrm{~W}_{2}=\mathrm{A}_{q p} \mathrm{~B}_{p f}$.

When the dimension of vector $p$ is more than that of vector $q$, the equation number of Eq.9 is less than that of Eq.1, the generalized displacement vector $q$ and velocity vector $\phi$ corresponding to $C$ elements can be determined by Eq.9 . Thus, the efficiency of system modeling and simulation can be increased.

When the dimension of vector $p$ is equal to that of vector $q$, the equation number of Eq.7 is equal to that of Eq.9. According to practical need, system state variables can be separated by Eq.7 or Eq.9, and the displacement vector $X_{I}$, flow vector $X_{I}^{\alpha}$ of inertia elements or the generalized displacement vector $q$, velocity vector $\phi$ corresponding to $C$ elements can be determined directly.

The procedure introduced above is organized and suitable for realizing on a computer by MATLAB[8]. Besides this, the system state space equations shown as Eq. 7 or Eq. 9 can be transformed into the first order differential equations, many numerical solving algorithm that are available can be used[1].

\section{Example system}

A spring mass system is shown in Fig.2, the masses of every mass block are $M_{1}, M_{2}, M_{3}, M_{4}$ and $M_{5}$, their displacements are $x_{I 1}, x_{I 2}, x_{I 3}, x_{I 4}$ and $x_{I 5}$, the corresponding velocities are $v_{I 1}, v_{I 2}, v_{I 3}, v_{I 4}$ and $v_{I 5}$. The stiffness coefficients of every spring are $k_{1}, k_{2}, k_{3}, k_{4}, k_{5}, k_{6}$ and $k_{7}$, their elastic displacements are $x_{1}, x_{2}, x_{3}, x_{4}, x_{5}, x_{6}$ and $x_{7}$, the corresponding velocities are $v_{1}, v_{2}, v_{3}, v_{4}, v_{5}, v_{6}$ and $v_{7}$. The force acting on mass block $M_{1}$ is $F(t)$, the end velocity of spring $k_{7}$ is $v(t)$, and the arms of force are $a$ and $b$ respectively. 


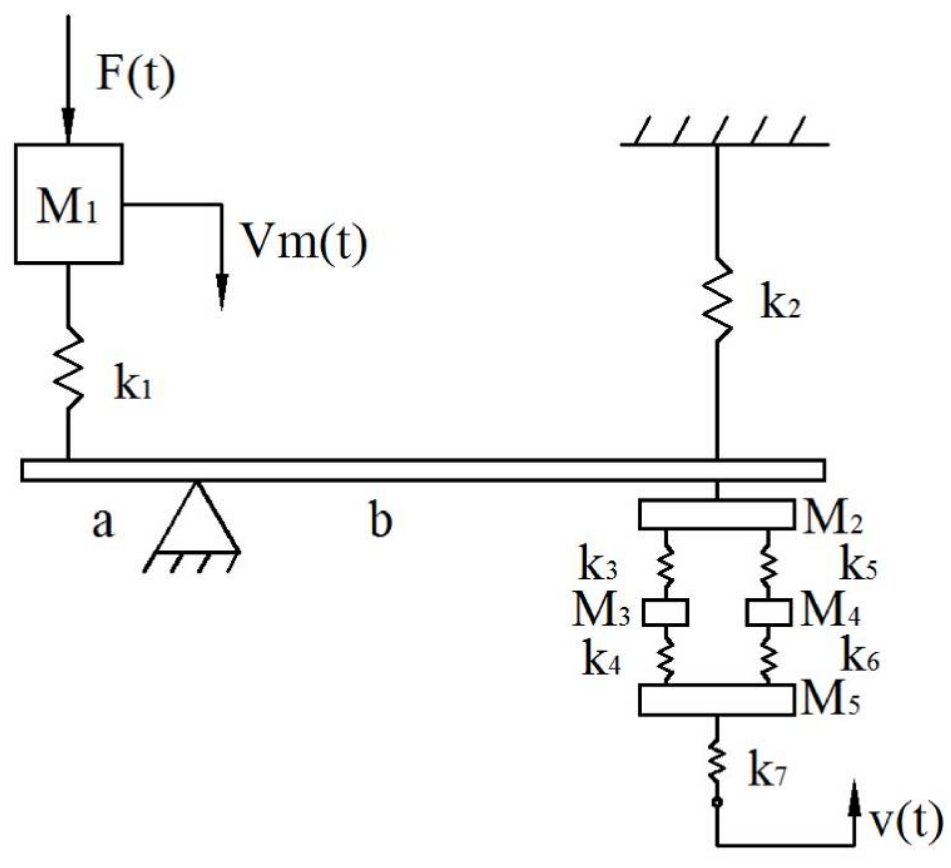

Fig.2 Mass spring system

From the system structure and the connecting relations of system components, the vector bond graph model of this mass spring system can be made and shown in Fig.3. By the procedure[3], the system state space equations as $X \mathcal{E}=A X+B U$ can be obtained. From Eq.1, the corresponding coefficient matrices are as following:

$$
\begin{aligned}
& \mathrm{A}_{p p}=\left[\begin{array}{ccccc}
0 & 0 & 0 & 0 & 0 \\
0 & 0 & 0 & 0 & 0 \\
0 & 0 & 0 & 0 & 0 \\
0 & 0 & 0 & 0 & 0 \\
0 & 0 & 0 & 0 & 0
\end{array}\right], A_{p q}=\left[\begin{array}{ccccccc}
-k_{1} & 0 & 0 & 0 & 0 & 0 & 0 \\
m_{1} k_{1} & -k_{2} & -m_{2}(1,1) k_{3} & -m_{2}(1,2) k_{5} & 0 & 0 & 0 \\
0 & 0 & k_{3} & 0 & -k_{4} & 0 & 0 \\
0 & 0 & 0 & k_{5} & 0 & -k_{6} & 0 \\
0 & 0 & 0 & 0 & m_{3}(1,1) k_{4} & m_{3}(1,2) k_{6} & -k_{7}
\end{array}\right], \\
& A_{q p}=\left[\begin{array}{ccccc}
\frac{1}{M_{1}} & -\frac{m_{1}}{M_{2}} & 0 & 0 & 0 \\
0 & \frac{1}{M_{2}} & 0 & 0 & 0 \\
0 & \frac{m_{2}(1,1)}{M_{2}} & -\frac{1}{M_{3}} & 0 & 0 \\
0 & \frac{m_{2}(1,2)}{M_{2}} & 0 & -\frac{1}{M_{4}} & 0 \\
0 & 0 & \frac{1}{M_{3}} & 0 & -\frac{m_{3}(1,1)}{M_{5}} \\
0 & 0 & 0 & \frac{1}{M_{4}} & -\frac{m_{3}(1,2)}{M_{5}} \\
0 & 0 & 0 & 0 & -\frac{1}{M_{5}}
\end{array}\right], A_{q q}=\left[\begin{array}{lllllll}
0 & 0 & 0 & 0 & 0 & 0 & 0 \\
0 & 0 & 0 & 0 & 0 & 0 & 0 \\
0 & 0 & 0 & 0 & 0 & 0 & 0 \\
0 & 0 & 0 & 0 & 0 & 0 & 0 \\
0 & 0 & 0 & 0 & 0 & 0 & 0 \\
0 & 0 & 0 & 0 & 0 & 0 & 0 \\
0 & 0 & 0 & 0 & 0 & 0 & 0
\end{array}\right], B_{p e}=\left[\begin{array}{c}
1 \\
0 \\
0 \\
0 \\
0
\end{array}\right], \\
& \mathrm{B}_{p f}=\left[\begin{array}{lllll}
0 & 0 & 0 & 0 & 0
\end{array}\right]^{T}, \mathrm{~B}_{q e}=\left[\begin{array}{lllllll}
0 & 0 & 0 & 0 & 0 & 0 & 0
\end{array}\right]^{T}, \mathrm{~B}_{q f}=\left[\begin{array}{lllllll}
0 & 0 & 0 & 0 & 0 & 0 & 1
\end{array}\right]^{T} \text {. }
\end{aligned}
$$




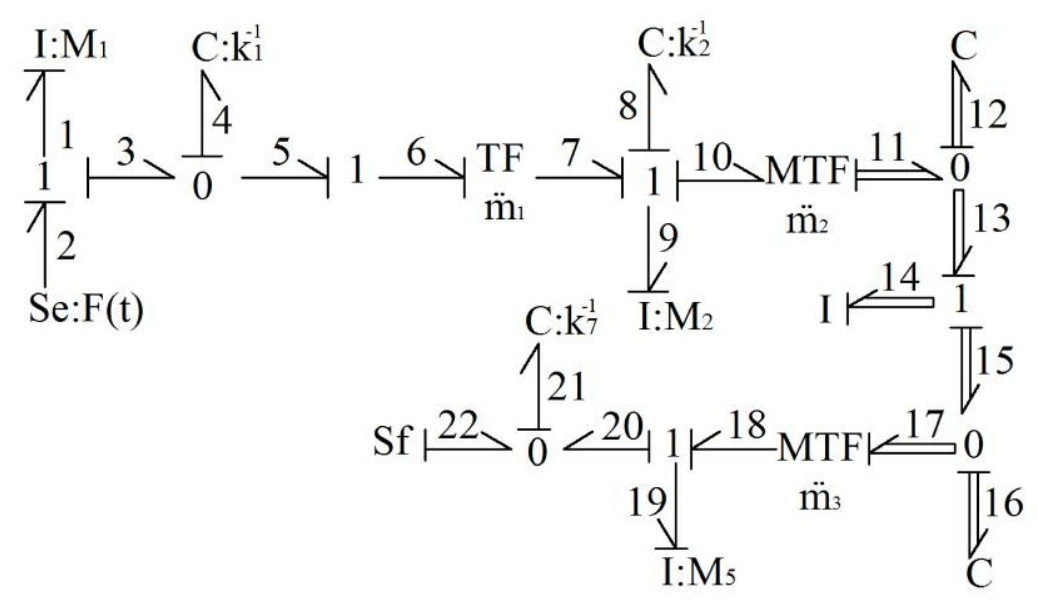

Fig. 3 System vector bond graph

For this system, the dimension of vector $p$ is less than that of vector $q$. Inputting the above matrices into the program based on MATLAB[8], the system dynamic equations in the form of Eq.7 can be obtained by the procedure presented here. Thus, the order reduction of system dynamic equations can be realized, and the final results are as following:

$$
\begin{aligned}
& {\left[\begin{array}{cccccc}
M_{1} & & & & \\
& M_{2} & & & \\
& & M_{3} & & \\
& & M_{4} & \\
& & & & M_{5}
\end{array}\right] \text { \& }_{I}+\left[\begin{array}{cccc}
k_{1} & -k_{1} m_{1} & 0 \\
-k_{1} m_{1} & k_{1} m_{1}^{2}+k_{2}+k_{3} m_{2}^{2}(1,1)+k_{5} m_{2}^{2}(1,2) & -k_{3} m_{2}(1,2) \\
0 & -k_{3} m_{2}(1,1) & k_{3}+k_{4} \\
0 & -k_{5} m_{2}(1,2) & 0 \\
0 & 0 & -k_{4} m_{3}(1,1)
\end{array}\right.} \\
& \left.\begin{array}{cc}
0 & 0 \\
-k_{5} m_{2}(1,2) & 0 \\
0 & -k_{4} m_{3}(1,1) \\
k_{5}+k_{6} & -k_{6} m_{3}(1,2) \\
-k_{6} m_{3}(1,2) & k_{4} m_{3}^{2}(1,1)+k_{6} m_{3}^{2}(1,2)-k_{7}
\end{array}\right] X_{I}=\left[\begin{array}{l}
1 \\
0 \\
0 \\
0 \\
0
\end{array}\right] F(t)+\left[\begin{array}{c}
0 \\
0 \\
0 \\
0 \\
-k_{7}
\end{array}\right] \int_{0}^{t} v(t) d t
\end{aligned}
$$

where, vector $\mathrm{X}_{I}=\left[\begin{array}{lllll}x_{I 1} & x_{I 2} & x_{I 3} & x_{I 4} & x_{I 5}\end{array}\right]^{T}$.

For this example, the Newton-Euler method [1] was used to derive the dynamic equations of this system, the results are in good agreement with that obtained by the procedure in this paper.

\section{Conclusions}

A vector bond graph procedure was presented for the order reduction of system dynamic equations. This procedure is very suitable for dealing with the systems containing the coupling of multi-energy domains. Compared with standard scalar bond graph, the procedure presented here is more compact and clear. According to the need of practical problem, the different form of order reduction formula can be derived by separating system state variables to realize the transformation of system dynamic equations. Besides these, this procedure is organized and very suitable for computer aided dynamic modeling and simulation. As a result, the efficiency of system dynamic analysis can be increased, and the application of vector bond graph method can be extended.

\section{Acknowledgements}

This work was financially supported by National Natural Science Foundation of China (Grant No. 51175272). 


\section{References}

[1] X. Z. Zheng, G. Y. Tang: Virtual Prototyping of Mechanical Systems(China Advanced Education Press, Beijing 2010)."In Chinese"

[2] Y. Y. Yang, D. W. Jin: Mechanical System Dynamics(Tsinghua University Press, Beijing 2009). "In Chinese"

[3] D. C. Karnopp, D. L. Margolis and R. C. Rosenberg: System Dynamics: Modeling and Simulation of Mechatronic Systems(John Wiley, New York 2000).

[4] T. Yu, Q. L. Zeng: Chinese Journal of Construction Machinery Vol.5, No.1(2007), p.10-15 "In Chinese"

[5] S. Behzadipour, A. Khajepour: Modelling Practice and Theory Vol.14(2006), p. 279-295

[6] Z. S. Wang., Y. Y. Tao: COMPUTER MODELLING \& TECHNOLOGIES Vol.18, No.9(2014), p. 497-502

[7] Z. S. Wang, Y. Y. Tao and Q. Y. Wen: Applied Mechanics and Materials Vol.321-324(2013), p. $1725 \sim 1729$

[8] J. F. Jiang, L. J. Hu and J. Tang: Numerical Analysisi and MATLAB Experiment(Science Press, Beijing 2004). "In Chinese" 